\title{
Congestion Avoidance for Multi-Hop Wireless Network
}

\author{
Nouhfa Juhiya S. ${ }^{1}$, Mrs. R. Divya ${ }^{2}$ \\ PG Scholar, EEE, PSG College of Technology, Coimbatore, India ${ }^{1}$ \\ Assistant Professor, EEE, PSG College of Technology, Coimbatore, India ${ }^{2}$
}

\begin{abstract}
Congestion is one of the major problem in Wireless Sensor Network which occurs when large number of nodes sending data to a common node. Because of this congestion, the node's buffer gets overloaded which either increases the packet service time or starts dropping the packets which in turn degrades the packet delivery ratio and also increases the node's energy consumption and end to end delay in the network. In healthcare applications, there is a need for continuous data transmission to the monitoring location and also the delay should be minimized. Also when the sensing parameters gets increased then the number of nodes in the network also gets increased, in such case the remote nodes cannot reach the central location in single-hop manner so there is a need for multi-hop forwarding. In order to overcome the above mentioned issues, multi-hop based congestion avoidance technique has been proposed. The objective of the proposed work is to avoid the congestion and thereby improving the performance metrics such as packet delivery fraction, average remained energy and average end to end delay. Initially performance analysis of different routing protocols like AODV, DSR and DSDV has been done, in order to select a best routing protocol for a multi-hop network. The topology used in the proposed work is clustered topology as multi-hop routing and it is evaluated in NS-2 simulator.
\end{abstract}

Keywords: Congestion avoidance; Wireless Sensor Network (WSN); multi-hop; routing protocols; clustered topology.

\section{INTRODUCTION}

Wireless Sensor Network is a set of sensor nodes which is used to monitor physical or environmental conditions and the data which is sensed is then processed, computed, aggregated and finally it is passed on to the main location called base station. The base station or sink node is the central location where the collected data from all other sensor nodes is stored and the data can be retrieved for future use [1]. The main advantages of WSN are the sensor nodes can be place in non-reachable areas such as deep forests, there is no need for any fixed infrastructure for a network setup, rural areas, etc., avoids wiring, supports node mobility and heterogeneous nodes, low cost for implementation and able to withstand even in wild environmental conditions [2].

The applications of WSN include tracking, environment monitoring, healthcare monitoring, landslide detection, traffic monitoring, forest fire detection, other natural disaster monitoring and etc. In WSN, the energy of nodes gets discharged based on the transmission and reception of both data and control packets. Since WSN is battery operated, in hostile environment the recharging of node's battery is not an easy task, so the energy consumption parameter is taken into account for most of the research for the improvement of the lifetime of the network [3]. So, in order to prolong the lifetime of the network the existing routing protocols should be selected efficiently for the type of network to be implemented. Since, routing protocols works optimally for certain network topologies.

WSN is used for many healthcare applications like monitoring the patient's bio parameters. In such applications there is need for continuous transmission of data to the monitoring location and here the congestion should be avoided while transmitting the data to the monitoring location. Since, congestion increases the delay and energy consumption. For medical applications, the delay should be greatly reduced since increased delay can lead to death of the patient.

\section{RELATED WORKS}

Literature survey say that transmission of large amount of traffic to a common destination node by multiple source nodes leads to congestion in wireless networks. This happens especially near the base station node, since the nodes that are closer to the base station will be transmitting more data packets thereby increasing its traffic burden. Conventionally, single-hop topology has been used to avoid congestion. But for a large network with more number of sensor nodes, the remote nodes cannot reach the central location in single hop due to its limited transmission range so there is a need for multi-hop forwarding. 


\section{A. Congestion in Wireless Sensor Network}

Jilani Sayyad and Dr.N.K. Choudhari [4] gave an overview about the types of congestion in WSN. There are two types of congestion in WSN which are node level congestion and link level congestion. The node level congestion arises when the input buffer or output buffer of node is overloaded which results in dropping the packets and increased queuing or processing delay. Because of the packet loss in node level congestion which leads to retransmission of packets thereby it consumes additional amount of energy. Link level congestion is caused when several active sensors in the network try to access the channel at the same time since wireless channels are shared by multiple nodes by using carrier sense multiple access (CSMA) protocol. This second type of congestion reduces both effective utilization of the link and overall throughput and also increases the packet service time. So this type of congestion also consumes additional energy. Therefore both node level congestion and link level congestion directly affects the energy consumption of nodes. Normally in wired networks when there is a need for transmitting more data packets to a single destination and also to reduce the congestion, one way is to increase the bandwidth whereas this is not possible in wireless networks because in some cases the sensor nodes will be following different IEEE standards. In such cases, hindrance may happen between the node following 802.11 standard and the node following 802.15.4 standard [5].

\section{B. Routing Protocols}

Routing protocols are used to disseminate the data from source node to the destination node. The routes can be constituted and maintained in sensor nodes either proactively or reactively. Anu Arya and Jagtar Singh [6] discuss the difference between proactive and reactive routing protocols. In proactive routing the routing table is distributed throughout the network periodically so that new lists of destinations and their paths from source to destination can be retained at regular time intervals. In this type of routing, the routing information such as path between the source to destination is computed and distributed before the actual data transfer. DSDV is one of the example of proactive routing. In reactive routing the path between source node and destination node is found only on demand. It does not compute and share the path before the actual data transfer unlike proactive routing. In this type of routing, the node which has data to send floods the network with route request control messages and the most immediate and relevant neighbor issues a route reply control messages to the request it received. This process continues until the destination node is reached in order to find the path. AODV and DSR are the examples of reactive routing.

\section{1) Ad hoc On- Demand Distance Vector (AODV):}

AODV routing protocol belongs to reactive routing, in such protocols there is no need to update the routing tables at regular interval of time unless there is any demand for new routes in the network. It supports unicasting as well as multicasting. If any source node has data to transmit to the destination node but it does not know the path between them, then in such case before making actual transmission of data it undergoes route discovery process. After the route discovery, the path found is maintained. For route discovery and route maintenance, it uses three type of control messages which are route request (RREQ), route reply (RREP) and route error (RERR). This RREQ message is broadcast by the source node throughout the network in which the destination number is mentioned to find the destination node [7]. The RREQ message is received at each intermediate node and a path to the source node is created. If the receiving node is not destination node then it rebroadcasts the RREQ message until the destination is reached. Once the RREQ message reaches the destination node by matching its destination sequence number, then it generates a RREP message. This RREP is propagated in a hop-by-hop manner to the source node through the same path by which destination is traced. This is how the source node computes the route to the destination and starts the data transmission. If the source receives more than one RREP messages, then it chooses the path which has shortest hop count to reach the destination. When there is a link failure in the network is detected, RERR control message is propagated towards the source and that route is invalidated and initiates new route discovery process if required.

2) Destination Sequenced Distance Vector (DSDV):

DSDV routing protocol belongs to proactive routing which gives improvement to the classical Bellman-Ford algorithm. Here all the nodes in the network maintains a routing table and these routing tables are distributed to their immediate neighbors periodically and also if there is any modification has found from the previous update. The routing table sent by a node includes sequence number, all available destination address and the number of hops to reach the destination node [7]. Here the sequence number assigned by the destination node is used to discover the stale routes from fresh routes and it helps to avoid loop formation. There are two types of routing table update, they are full dump and incremental update. In full dump, all available routing information is sent to the neighbor nodes whereas in an incremental update it sends only the modified metric in the routing table since the previous update.

\section{3) Dynamic Source Routing (DSR):}

DSR belongs to the category of reactive routing protocol which initiates route discovery only on demand similar to AODV. DSR provides source routing feature and this feature brings the main difference in AODV and DSR and thus resulting in varied performance levels. DSR stores the complete path from source to destination including the 
intermediate nodes through which it reaches the destination in its routing table whereas AODV stores only the next hop node in its routing table. So the information about the path is listed in the packet header by the packet sender to reach its destination [8]. This form of routing is known as source routing. Similar to AODV, DSR has a pair of control messages to discover the path from source to destination. For route discovery, it uses RREQ and RREP control messages. The RREQ message is broadcasted in the network by the source node which has data to send to the specific destination node. The neighbor nodes receiving the RREQ message will add its address and then rebroadcast that RREQ message. When this message reaches the specified destination then that is the route from source to destination. During route discovery process, if the message did not reach the destination, then before rebroadcasting the RREQ control message, the node will check for whether there is any previous route to a specified destination in the route cache. This is how memory overhead is reduced by maintaining the route cache. The route maintenance mechanism is only used when the source node is actually sending packets to the destination node.

\section{PROPOSED WORK}

To avoid the congestion in wireless network for continuous data transmission and also for a large network topology, the multi-hop clustered topology is proposed. Moreover, compared to single wireless links, the proposed multi-hop wireless links have several benefits such as network coverage is extended, availability of several paths which improves the robustness of the network and also the requirement of the transmission power for several short links is less than the single long link. Fig. 1 shows the clustered topology as multi-hop routing. For a multi-hop connectivity, there are one or more intermediate nodes along the path from source to destination which receive data and forward through wireless links. Here routing protocol is used to find the efficient path from source to destination. So, in order to select a good routing protocol for a multi-hop network, initially the performance analysis of proactive and reactive routing protocols has been performed for the parameters such as energy consumption, packet delivery, delay and total number of hops.

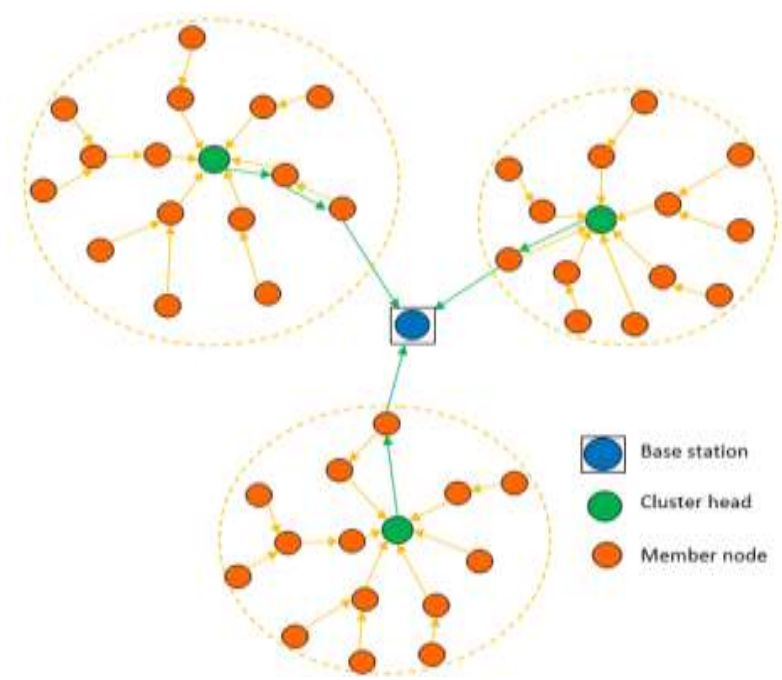

Fig. 1. Multi-hop clustered topology

A. Assumption model

- Static clustering topology as multi-hop routing is used

- In static clustering, initially nodes are placed in the clustered form

- All the nodes have fixed position, so it has fixed $\mathrm{x}$ and $\mathrm{y}$ coordinates

- In each cluster, the cluster head $(\mathrm{CH})$ is selected.

- The node which has the maximum energy and also minimum distance to all other nodes in the cluster is selected as the cluster head $(\mathrm{CH})$

- $\mathrm{CH}$ collects the data from its member nodes and the aggregated data is forwarded to the base station

- For a multi-hop clustering topology, the member nodes in the cluster chooses an optimal path to reach its $\mathrm{CH}$ and also the aggregated data is transmitted through an optimal path from $\mathrm{CH}$ to the base station.

\section{B. Network Model}

The simulation tool used to execute the proposed work is NS-2 simulator version 2.35 and the platform used to run the NS-2 is Ubuntu 12.04. Network simulator is an open source discrete event simulator which covers wide applications of different protocols for various types of networks with different traffic models and other network parameters. Using network simulator, the validation of network design for wireless sensor networks can be done in cost effective manner. 
$\mathrm{C}++$ and OTcl are the two main languages used in network simulator [9]. $\mathrm{C}++$ helps in improving the simulation efficiency, provide information about the protocols and their operation. OTcl is the object oriented variant of Tool Command Language which helps to define protocols and its applications and also various network topologies can be described. In network simulator, the simulation is followed by an animation tool called Network Animator (NAM) which displays the working of the protocols in the network.

1) Simulation setup 1:

For analysing the performance of routing protocols such as AODV, DSR and DSDV, the simulation parameters are used as shown in Table I. The variation in the performance of routing protocols can be analysed by increasing the number of nodes for the same simulation parameters. The analysis is done for continuous transmission, so all nodes in the network starts transmitting the data to the common sink node. For this type of continuous transmission and for a multi-hop network, the performance metrics such as packet delivery fraction, average remained energy, average end to end delay and total number of hops are analysed.

TABLE I SIMULATION PARAMETERS

\begin{tabular}{|l|l|}
\hline Parameter Type & Parameter Value \\
\hline Number of nodes & $15,25,35$ \\
\hline Queue type & Priority Queue \\
\hline Routing protocols & AODV, DSR, DSDV \\
\hline Traffic model & CBR \\
\hline Initial energy & 100 Joules \\
\hline Network dimension & $8000 \mathrm{~m} * 1000 \mathrm{~m}$ \\
\hline Platform & Ubuntu \\
\hline Simulator & NS-2.35 \\
\hline
\end{tabular}

2) Simulation setup 2:

The simulation parameters used for multi-hop clustering topology is shown in Table II. In static clustering, initially nodes are deployed in the clustered form. Totally forty-two nodes are deployed in a static clustered form in which three nodes are selected as cluster head and one node is selected as sink node. The sink node is placed at the center, so that the energy consumed to reach the sink node can be minimized. In each cluster, the cluster head is selected based on the energy and distance. The node which is having the maximum energy and minimum distance to reach all its member nodes and sink node is elected as cluster head.

TABLE II SIMULATION PARAMETERS

\begin{tabular}{|l|l|}
\hline Parameter Type & Parameter Value \\
\hline MAC layer & 802.11 \\
\hline Routing protocol & DSR \\
\hline Number of nodes & 42 \\
\hline Number of cluster heads & 3 \\
\hline Queue type & Priority Queue \\
\hline Traffic model & CBR \\
\hline Packet size & 210 Bytes \\
\hline Initial energy & 100 Joules \\
\hline Transmission range & $250 \mathrm{~m}$ \\
\hline Network dimension & $8000 \mathrm{~m} * 1000 \mathrm{~m}$ \\
\hline Platform & Ubuntu \\
\hline Simulator & NS-2.35 \\
\hline
\end{tabular}

\section{SIMULATION RESULTS AND ANALYSIS}

In this section, the results for the performance analysis of routing protocols such as AODV, DSR and DSDV and also the results for multi-hop clustered topology for avoiding congestion is discussed.

For simulation setup 1, the performance evaluation is based on the simulation parameters as shown in Table I. Four key performance metrics such as packet delivery fraction, average remained energy, average end to end delay and total number of hops are considered for evaluating the performance. Packet delivery fraction or PDF is the ratio of the number of data packets successfully delivered to the destination nodes to the data packets sent by the source nodes. The packet delivery fraction must be greater for the better performance of the protocol. 


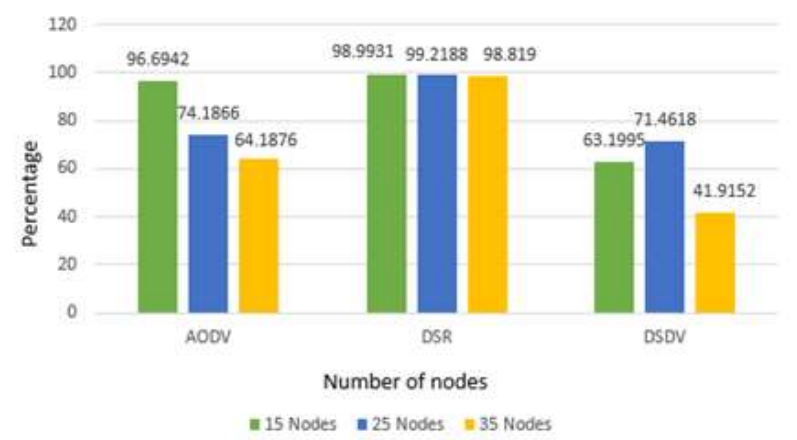

Fig. 2. Performance Analysis of Packet Delivery Fraction

Fig. 2 shows that the PDF of AODV gets reduced as the number of nodes in the network increases whereas in DSR the PDR is maintained greater even when the number of nodes gets increased. But in DSDV the packet delivery is very less since the generated packets are not sent to the destination for a multi-hop network which is analysed in the NAM window. Thus, DSR has the maximum packet delivery fraction for a multi-hop wireless network.

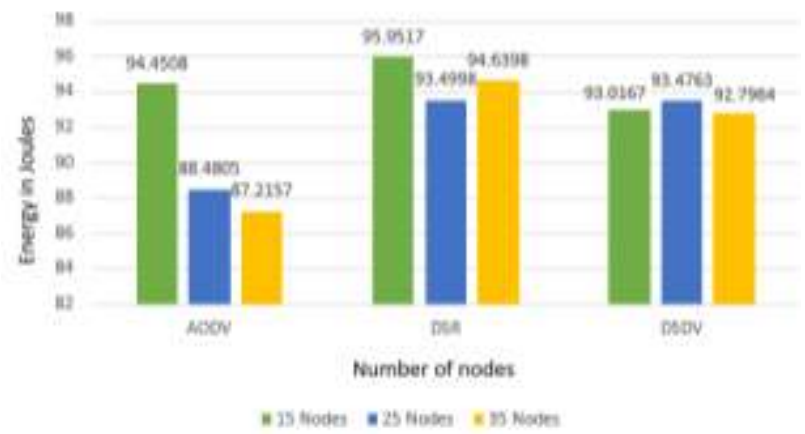

Fig. 3. Performance Analysis of Average Remained Energy

Average remained energy is the ratio of total residual energy in all the nodes in the network to the total number of nodes in the network. Fig. 3 shows that the average remained energy of AODV gets reduced as the number of nodes in the network increases and in DSR the average remained energy is greater even for increasing number of nodes and in DSDV also the average remained energy is greater since most of the nodes in the multi-hop network did not transmits its data packets and so the energy of most of the nodes is not utilized. But DSR is capable of forwarding the maximum packets and its average remained energy is also maintained higher.

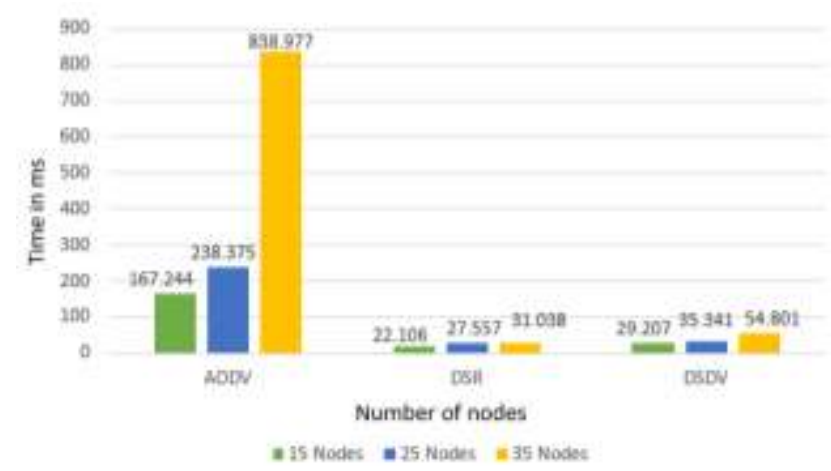

Fig. 4. Performance Analysis of Average End to End Delay

Average end to end delay is the total time taken for the transmission of packets from source to destination across the network including route discovery process, queuing, processing and propagation delay. Fig. 4 shows the performance analysis of average end to end delay. The average end to end delay of AODV increases greatly for more number of nodes whereas in DSR and DSDV the average end to end delay is very much reduced compared to AODV.

Total number of hops is the total hops the packets are forwarded across the network from source to destination. In multi-hop networks, the total number of hops can be reduced by selecting the shortest route from source to destination. Fig. 5 shows the performance analysis of total number of hops. As discussed earlier, from the analysis of NAM window most of the nodes did not transmit the data packets to the destination for a multi-hop network in DSDV and only few 
nodes send its data and therefore it took less number of hops. But both AODV and DSR forwards the data to the destination. So, when comparing its total number of hops DSR has less compared to AODV.

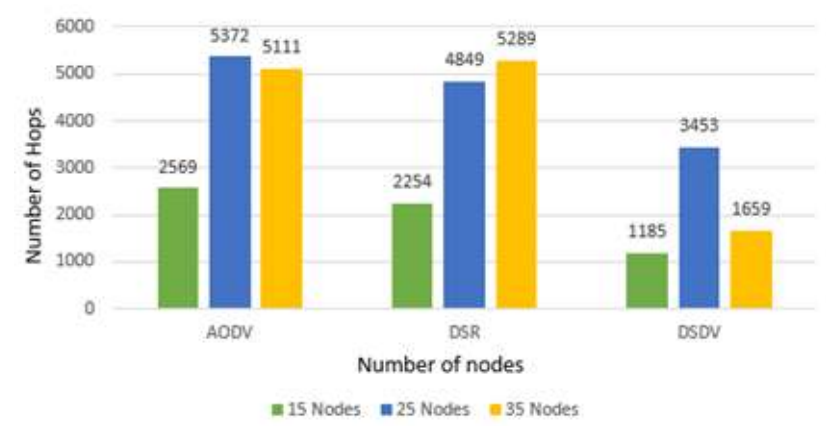

Fig. 5. Performance Analysis of Total Number of Hops

The overall performance of DSR gives better results for a multi-hop wireless network compared to AODV and DSDV. So DSR routing protocol is used in multi-hop clustered topology.

TABLE III COMPARISON OF MULTI-HOP AND SINGLE-HOP CLUSTERED TOPOLOGY WITH REMOTE NODES

\begin{tabular}{|l|l|l|}
\hline Performance Metrics & $\begin{array}{l}\text { Multi-Hop Clustered Topology with } \\
\text { Remote Nodes }\end{array}$ & $\begin{array}{l}\text { Single-Hop Clustered Topology with } \\
\text { Remote Nodes }\end{array}$ \\
\hline Packet Delivery Fraction & $88.9099 \%$ & $76.7506 \%$ \\
\hline Average Remained Energy & 94.009 joules & 89.651 joules \\
\hline Average End to End Delay & $25.5977 \mathrm{~ms}$ & $27.4913 \mathrm{~ms}$ \\
\hline
\end{tabular}

Table III shows the comparison of results of multi-hop and single-hop clustered topology with the presence of remote nodes in the network. The comparison result shows that the packet delivery fraction for multi-hop gives better results compared to single-hop when there are presence remote nodes in the network. Also multi-hop gives improved results for average remained energy and average end to end delay when compared to single-hop. Thus, the performance of multi-hop clustered topology gives improved results and it is suitable for a wireless network with remote nodes.

\section{CONCLUSION}

In this work, multi-hop based congestion avoidance method is proposed which avoids the congestion problem along with energy consumption reduction for a multi-hop wireless network. The simulation results of performance analysis of proactive and reactive routing protocols have shown that the overall performance of DSR routing protocol gives better results than AODV and DSDV. Thus, DSR protocol is suitable for multi-hop wireless networks. Also by using this efficient routing protocol in multi-hop clustered topology gives better results with respect to average remained energy, PDR, end to end delay and total number of hops.

In the future scope, the proposed work can be extended for a dynamic clustering topology for both single-hop and multi-hop routing. Also for a topology using mobility model, an efficient routing protocol should be determined.

\section{REFERENCES}

[1] Zhang, Hongwei, Anish Arora, Young-ri Choi, and Mohamed G. Gouda, "Reliable bursty converge cast in wireless sensor networks", Computer Communications 30, no. 13, pp.2560-2576, 2007.

[2] K. Sarammal, R. A. Roseline, "A Review: Wireless Sensor Networks and Its Application, Platforms, Standards and Tools", International Journal of Computer Trends and Technology (IJCTT), vol. 4 ,August 2013.

[3] Md. Zair Hussain, M. P. Singh and R. K. Singh, “Analysis of Lifetime of Wireless Sensor Network”, International Journal of Advanced Science and Technology, vol. 53, April, 2013

[4] Jilani Sayyad and Dr.N.K. Choudhari, "Congestion Control Techniques in WSN and Their Performance Comparisons", International Journal of Multidisciplinary and Current Research, vol.3, February 2015.

[5] Jasleen Kaur, Kamaljit Singh Saini, Rubal Grewal, "Priority Based Congestion Avoidance Hybrid Scheme for Wireless Sensor Network", 1st International Conference on Next Generation Computing Technologies (NGCT-2015) Dehradun, India, 4-5, September 2015.

[6] Anu Arya, Jagtar Singh,"Comparative Study of AODV, DSDV and DSR Routing Protocols in Wireless Sensor Network Using NS-2 Simulator", (IJCSIT) International Journal of Computer Science and Information Technologies, vol. 5, no. $4,2014$.

[7] B.N. Jagdale, Pragati Patil, P. Lahane, D. Javale, "Analysis and Comparison of Diatance Vector, DSDV and AODV Protocol of MANET", International Journal of Distributed and Parallel Systems (IJDPS), vol. 3, no. 2, March 2012.

[8] Rakesh Poonia, Amit Kumar Sanghi, Dr. Dharm Singh, "DSR Routing Protocol in Wireless Ad-hoc Networks: Drop Analysis", International Journal of Computer Applications, vol. 14, no.7, February 2011.

[9] An Introduction to NS, Nam and OTcl scripting, NUIM-CS-TR- 2004-05, April 2004. 